\title{
Workshop: Pen-and-Paper Role-Playing
}

\author{
Florian Berger and Alexander Marbach \\ Pädagogische Hochschule / University of Education Weingarten, Germany \\ $\{$ berger, marbach $\}$ md-phw. de
}

\section{Workshop Abstract}

Over the last 30 years, a phenomenon of human-to-human interactive storytelling has developed, known as fantasy role-playing games ( $R P G)$, beginning with the publishing of the game Dungeons $\&$ Dragons in 1974. Since then, a role-playing culture has emerged, incorporating concepts from theatre, board games, history reenactment and storytelling, remixed in an almost post-modern way.

A role-playing session is in its very nature an interactive storytelling session, and the game master usually solves problems similar to those involved in Interactive Digital Storytelling (IDS) systems.

The goal of this workshop is to offer researchers in IDS valuable insights into principles of pen-and-paper RPGs, in particular in strategies of the game master. This is why the authors bring such a session to the conference, and moreover involve workshop participants as active players. The workshop consists of the following parts:

Introduction: The basic concepts of a pen-and-paper RPG are explained: the game master as the creator and narrator of the virtual world; players as the leaders of their respective fictional characters; resolving conflicts using rulesets; rules of in-game communication; character creation.

Playing Session: This is the actual role-playing, with the players completing a quest created by the game master. He has prepared a set of non-player characters (NPCs), maps of some key locations and some key events, but the actual storyline emerges by player interaction over the course of the game. Typical RPG features include suspense, time pressure, riddles, teamwork, acting and conflicts/combat. There are some typical problems a game master has to face during gameplay, most of them related to the conflict between the players' freedom to act and the demand for a consistent storyline. A game master makes use of several techniques to tackle these challenges.

Meta Discussion: This serves the purpose of answering questions for firsttime players, but every participant is encouraged to give personal views and impressions and ask questions. Game mastering techniques like reacting towards unexpected developments are revealed and discussed. Possible implications of the game play for future research in interactive digital storytelling are summed up and discussed. 